ROCZNIKI HUMANISTYCZNE

Tom LXVIII, zeszyt 5 - 2020

DOI: http://dx.doi.org/10.18290/rh20685-13

KATARZYNA WOŁOWSKA

\title{
PERTURBATION DU CADRE TEMPOREL ET PARTICIPATIF DE L'INTERACTION SUR LES FORUMS DE DISCUSSION EN LIGNE
}

\begin{abstract}
Ré s u mé. L'article a pour but de décrire quelques perturbations du modèle traditionnel de l'interaction verbale dans le contexte des forum de discussion en ligne. L'analyse concerne les topics relevés sur des forums français, où l'interaction, une fois terminée, est reprise et poursuivie après une longue période (parfois après plusieurs mois ou années). Ce relancement est possible puisque le topic est ouvert indéfiniment, ce qui permet de se référer à n'importe quel post et à tout moment. Du point de vue théorique, cela affecte les paramètres de base de l'interaction traditionnellement comprise : son cadre temporel (extension de la durée de la discussion), sa structure (manque de formules de clôture) et sa configuration actantielle. Cette pratique, bien qu'évitable dans les interactions face-to-face, est naturelle et fréquente dans les discussions sur les forums en ligne.
\end{abstract}

Mots clés : interaction verbale ; forum de discussion ; Internet ; modèle de la communication verbale ; perturbation.

\section{LE DISCOURS D'INTERNET COMME OBJET DE LA RECHERCHE LINGUISTIQUE}

A l'heure actuelle, le discours d'Internet, se répandant depuis quelques décennies à la vitesse de la lumière, constitue un objet de la réflexion linguistique de plus en plus attrayant, tant du point de vue descriptif que théorique. L'analyse de nouveaux types de discours produits dans le Réseau permet d'observer une sérieuse modification du modèle de la communication qui relativise et met en cause bien des paramètres considérés comme stables encore il y a une cinquantaine d'années. Les études consacrées à ce corpus

Dr habil. KATARZYNA WOŁOWSKA - professeur à l'Université Catholique de Lublin Jean-Paul II, Faculté des sciences humaines, Institut de linguistique, Chaire de linguistique romane ; adresse pour correspondance : Aleje Racławickie 14, 20-950 Lublin; courriel : wolowska@kul.pl. ORCID: https://orcid.org/0000-0003-2931-0689. 
riche en nouvelles formes linguistiques (p. ex. Mourlhon-Dallies, Rakotonoelina et Reboul-Touré ; Marcoccia ; Colin et Mourlhon-Dallies ; Münchow ; Pierozak ; Francq etc.) signalent et abordent différents problèmes liés soit à la structure des discours créés sur Internet, soit à leur nature générique (nouveaux genres inédits), soit aux facteurs pragmatiques qui déterminent leur portée communicationnelle.

Dans ce contexte pluriaspectuel de la communication menée on-line, il est intéressant d'observer le succès des forums de discussion. Bien qu'à présent l'intérêt porté à ceux-ci par les usagers d'Internet décroisse visiblement en faveur des réseaux sociaux (Facebook, Twitter, Instagram etc.), ces espaces virtuels de rencontre et d'échange d'idées sont toujours vivants et concentrent un public toujours important. Du point de vue linguistique, les textes produits sur les forums, à caractère par principe polylogal (sur le polylogue, $c f$. p. ex. Cabasino ; Traverso), constituent un champ d'exploration particulièrement intéressant vu leur nature non-univoque, unissant d'une manière créative les paramètres des modes de discours oral et écrit ( $c f$. Wołowska). En fait, l'interaction menée sur un forum de discussion, sans être libre de contraintes et de défauts (tels que l'anonymat et le mode indirect du contact qui bloque l'accès aux données communicationnellement cruciales comme la mimique, le geste ou l'intonation de l'interlocuteur), ouvre des possibilités de structuration formelle exclues dans le cas de l'échange verbal en direct.

Comme le remarque Marcoccia, la recherche sur ce corpus «[repose] souvent sur une démarche qui consiste à appliquer les catégories habituelles de l'analyse conversationnelle (définies pour l'étude des interactions en face-à-face) aux échanges médiatisés par ordinateur afin d'en identifier les spécificités » (1). Il est en effet intéressant d'observer les modifications que le mode du contact entre les interactants est susceptible d'effectuer dans la structure et la nature de la conversation.

L'objectif du présent article est de décrire quelques perturbations du modèle typique de la communication dans la discussion sur des forums d'Internet, qui résultent de l'extension excessive du cadre temporel de l'interaction. Pour ce faire, nous nous appuyons sur l'analyse d'une trentaine de topics développés sur des forums de discussion français, généraux ou spécialisés, où le phénomène qui nous intéresse se laisse observer d'une manière plus ou moins nette. 


\section{LE CADRE TEMPOREL DE L'INTERACTION MENÉE SUR UN FORUM DE DISCUSSION ON-LINE}

Si l'on compare les paramètres formels de l'interaction qui se déroule en direct et ceux de tout type de dialogue développé indirectement, surtout sous forme écrite (échanges de lettres, d'e-mails, de sms, de commentaires sur les sites d'Internet, de posts sur les forums etc.), l'une des différences immédiatement observables concerne la dimension temporelle où les deux types d'interaction peuvent s'inscrire selon le cas. Par principe, dans le cas la discussion " traditionnelle », les participants se rencontrent pour interagir dans un même cadre spatio-temporel (cela vaut même pour la vidéo-conférence où l'unicité de ce cadre est assurée par des moyes technologiques adéquats), alors que l'échange de messages indirect admet une extension plus ou moins importante de l'espace et du temps où il se déroule.

En effet, dans le cas qui nous intéresse ici, celui des forums de discussion, une réponse ou un enchaînement quelconque sur ce qui a été déjà dit dans le sujet (topic) donné peut s'ajouter à n'importe quel moment, indépendamment de la disponibilité des autres participants de la discussion. Pour l'illustrer, considérons l'exemple (1) qui vient du forum www.mamanpourlavie.com/forum/ (sujet intitulé Je ne suis pas une bonne mère) où, sans focaliser notre attention sur le contenu (disponible sous le lien fourni), nous résumons les paramètres qui nous intéressent, à savoir l'ordre des posts, les nicks des intervenants et, surtout, la date et l'heure de la publication des posts :

(1) Sujet Je ne suis pas une bonne mère, lancé le 4 novembre 2013.

Usager / date de la publication : astragale / posté le: 4 novembre 2013 20:40:30

Mumy / posté le: 5 novembre 2013 10:55:58

BoneMary/posté le: 5 novembre 2013 20:31:29

cocotte66 /posté le: 5 novembre 2013 21:23:51

mariekiki / posté le: 6 novembre 2013 17:31:06

mimimilou / posté le: 9 novembre 2013 10:36:15

Skit / posté le: 10 novembre 2013 10:33:30

mimimilou / posté le: 10 novembre 2013 15:09:12

mariekiki / posté le: 10 novembre 2013 18:49:26

astragale / posté le: 11 novembre 2013 13:23:28

mariekiki / posté le: 11 novembre 2013 17:08:43

Skit / posté le: 11 novembre 2013 17:17:25

Croustille44 / posté le: 11 novembre 2013 20:48:00

marisa1988 / posté le: 21 novembre 2013 02:11:36 
Lalionne85 / posté le: 21 août 2014 03:42:08 mathé / posté le: 4 mai 2015 23:50:42 mjplouffe / posté le: 17 octobre 2015 11:59:18 Pazouzou / posté le: 22 mai 2016 20:30:47 Hanane74 / posté le: 8 août 2017 02:46:28 marielovesimon / posté le: 25 novembre 2018 11:07:47

(http://www.mamanpourlavie.com/forum/sujet/je-ne-suis-pas-une-bonne-ma-re, consulté le 29 janvier 2020)

Si l'on regarde les dates de la publication des posts, on se rend compte très vite que la discussion, qui est assez courte, s'étend considérablement dans le temps: le post d'astragale qui inicie le topic date du 4 novembre (20h40) et la première réponse apparaît le 5 novembre à $10 \mathrm{~h} 55$. Ensuite, après deux heures d'intervalle, vient le post suivant, et puis 4 jours de silence sur le forum avant que mimimilou apparaisse et relance la discussion. Ce qui est intéressant, c'est que le premier intervenant (l'auteur du topic : astragale) ne participe pas dans la discussion, elle publie son premier post et elle ne répond pas, mais la négociation se déroule, tout le monde s'adresse à elle et discute aussi avec les autres pendant son absence. Or, astragale revient après une semaine (le 11 novembre) pour apporter de ses nouvelles et pour commenter ce que les autres lui ont répondu pendant cette semaine. Encore trois réponses apparaissent le même jour et la conversation semble terminée, d'autant plus que dix jours plus tard, le 21 novembre, intervient une modératrice (marisa1988) qui supprime une des réponses en tant que non conforme aux règles d'utilisation du forum.

Même si la discussion s'étend déjà considérablement dans le temps (14 répliques échangées en 17 jours), un tel rythme de la publication des posts ne présente rien de particulier vu qu'elle s'enferme dans l'espace du même mois (novembre 2013). Par contre, les posts suivants enfreignent déjà fortement les normes de l'interaction standard : le post de Lalionne85 est publiée le 21 août 2014, c'est-à-dire neuf mois après la clotûre apparente de l'interaction, et il ne suscite pas de discussion. Il en va de même des posts suivants qui se trouvent publiés avec des pauses de 5 à 15 mois (deux posts en 2015 et seulement un respectivement en 2016, 2017 et 2018). Certains usagers s'en rendent compte d'ailleurs; par exemple, mjplouffe qui publie dans le sujet le 17 octobre 2015 commence son post par la remarque : Je sais que le message date mais je ne pouvais m'empêcher de répondre... Pourtant, d'autres semblent ne pas avoir regardé la date de la publication des posts précédents, telle Pazouzou qui, en publiant son post le 22 mai 2016, cite celui 
de mimimilou datant du 9 novembre 2013 et enchaîne bel et bien sur son contenu. Le dernier post, au moment où nous avons consulté le forum (29 janvier 2020), date du 25 novembre 2018, mais le topic n'est pas clos et on ne saurait garantir qu'il se sera pas repris dans l'avenir.

Abstraction faite des détails, cela veut dire que la discussion sur un forum actif (à condition que le sujet ne soit pas fermé par les modérateurs) n'est jamais terminée définitivement, mais elle peut être toujours relancée, même plusieurs semaines, mois ou années après le «dernier » post. Une telle situation est plus fréquente qu'on ne l'aurait pensé : il arrive bien souvent qu'une discussion lancée et développée à un moment donné se trouve abandonnée pour un certain temps et ensuite reprise, relancée et enrichie par de nouveaux arguments et/ou points de vue (souvent, l'usager qui relance le topic, enchaîne sur le premier post, celui où le problème se trouve exposé). Ainsi, surtout dans les cas des sujets qui abordent les problèmes universels, bien que l'interaction soit "vieille», elle n'est pratiquement jamais close. La possibilité de relancer une telle discussion (ou, comme le disent les usagers des forums eux-mêmes, de la « déterrer $»^{1}$ ) la distingue nettement d'une interaction menée en direct qui, une fois terminée, ne peut pas être reprise en tant que telle, mais seulement les problèmes qu'elle aborde peuvent être rediscutés dans une nouvelle interaction.

Si l'on considère cette démarche du point de vue de la structure de l'interaction, les topics qui ouvrent la possibilité de relancement de la discussion présentent des caractéristiques communes. Ainsi, l'interaction commence habituellement par une exposition du problème (ce qui est une pratique standard sur les forums), accompagnée ou non par des termes d'adresse aux destinateurs (ou groupes de destinateurs) préférés par l'usager qui initie le sujet ; cependant, la discussion ne se termine pas par des formules de clôture typiques, mais elle est rompue in medias res, ce qui favorise naturellement sa reprise à n'importe quel moment.

Bien entendu, une telle pratique n'est pas tout à fait neutre; pour cette raison, il arrive que les discutants, au lieu d'enchaîner sur le contenu du post qui relance le sujet, font des remarques métadiscursives de type :

(2) OSSE : Pour davantage de clarté, il est préférable de créer un nouveau post que de reposter dans un vieux sujet pour présenter son vélo.

(https://forum.tontonvelo.com/viewtopic.php?f=46\&t=29498).

\footnotetext{
${ }^{1}$ Il y existe même des glossaires de forums où l'on peut trouver une définition comme celle-ci : «Déterrage : lorsque quelqu'un intervient dans un vieux sujet et le relance» (http://forumauto.caradisiac.com/aide/FAQ-Tutoriaux-et-guides-pratiques/sujet7.htm).
} 
(3) Théocrite : Il aurait été préférable te créer ton propre sujet plutôt que de répondre dans un vieux sujet qui d'après moi ne correspond pas à ton problème

(https://forums.cnetfrance.fr/topic/116508-comment-supprimer-un-site/).

Il y a même plus : parfois, le phénomène qui nous intéresse finit par susciter une discussion dans un sujet à part (cf. les exemples 3-4).

(4) k0xid (dans : Déterrer un vieux sujet) :

Salut à tous, je voulais savoir, pourquoi est ce que c'est mal vu de répondre à un vieux sujet dans les forums? (parfois c'est carrément interdit).

je suis pas très actif dans les forums (je suis plus du genre à chercher sur google pour tomber sur des topics similaires à mon problème) du coup je me demande pourquoi on aime pas déterrer les sujets (n'est ce pas mieux de répondre à un sujet déjà existant plutôt que de faire un doublon ?)

(http://www.jeuxvideo.com/forums/42-51-59203262-1-0-1-0-deterrer-un-vieuxsujet.htm).

(5) XiaoGe (dans : Déterrer un vieux sujet / Créer un nouveau sujet)

Bonjour à tous. En parcourant le forum, je suis tombé sur un topic qui avait été déterré. Un membre très actif du forum, donc je pense qu'on peut se fier sans réserve à ce qu'il/elle dit concernant le fonctionnement du forum, a précisé dans sa réponse qu'il valait mieux créer un nouveau sujet plutôt que d'en déterrer un.

Je me pose alors la question suivante (...) : Pourquoi vaut-il mieux créer un nouveau sujet, même si le même existe déjà, mais inactif depuis quelques mois ? Ces doublons ne sont pas gênants ? Avec un peu de chance, notre membre très actif se reconnaîtra et c'est avec plaisir et intérêt que je lirai sa réponse ;)

Aloxxx :

Sinon une possible raison c'est que lorsqu'un sujet est déterré, certains vont répondre ensuite sans regarder à des propos vieux d'il y a plusieurs mois, et souvent obsolètes. Dont souvent les auteurs ne sont plus présents ou plus actifs. Et ça crée des monologues sans aucun sens.

(https://www.hearthstone-decks.com/forum/suggestions-et-bugs/deterrer-unvieux-sujet-creer-un-nouveau-sujet-t98190).

Les exemples cités ci-dessus signalent clairement que « déterrer » un sujet non actif depuis un certain temps est une pratique certes marquée par rapport aux normes communicationnelles, mais, en définitive, traitée comme acceptable. Il est à noter que, dans l'exemple (3), Théocrite est modéractrice $\mathrm{du}$ forum dont le rôle est d'assurer au sein de celui-ci l'ordre et la lisibilité des topics, ce qui explique sa remarque métadiscursive. Dans les autres 
exemples, ce sont les usagers qui abordent eux-mêmes le problème des sujets déterrés du moment où cette démarche attire leur attention.

Le phénomène de relancer la discussion après une rupture temporelle suscite des questions quant à la clôture définitive d'une telle discussion. En effet, n'existerait-il plus de limites d'une telle extension temporelle de l'interaction? Ou bien s'agit-il seulement de plusieurs conversations à part, « collées » artificiellement au sein d'un même topic ? A quel point peut-on faire abstraction de la cohérence thématique du sujet lancé, développé, abandonné pour longtemps et puis relancé ? Comme il est difficile de trancher si l'on reste sur le plan structural et sémantique, c'est sur le plan pragmatique qu'il faut chercher des facteurs pertinents. En fait, la réponse d'Aloxxx dans l'exemple (5) signale que la reprise d'un vieux sujet perturbe (ou du moins risque de perturber) un aspect fondamental de l'interaction, à savoir sa configuration actantielle.

\section{LES ACTANTS DE LA DISCUSSION : PERTUBATIONS DU CADRE PARTICIPATIF}

Rappellons ici un schéma bien connu (Kerbrat-Orecchioni 86) qui englobe et systématise différents types d'actants de l'interaction au sein de ce qu'on appelle son cadre participatif (participation framework, cf. Goffmann).

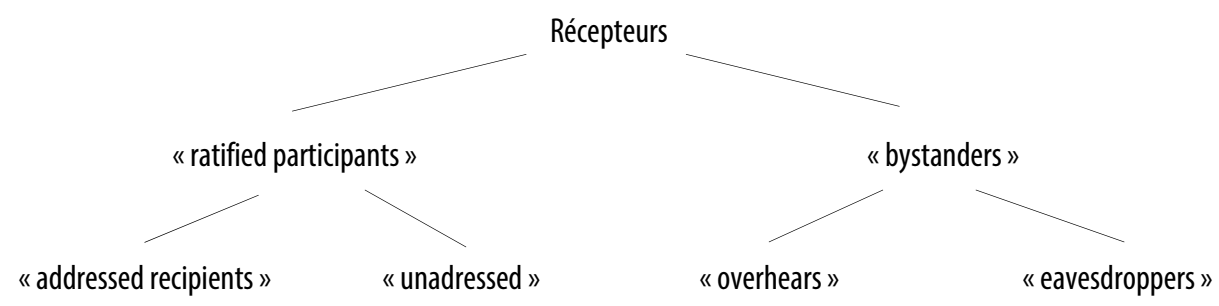

Schéma 1 : Cadre participatif de l'interaction (Kerbrat-Orecchioni 86).

En fait, le statut des participants de la conversation n'est pas univoque : à côté des actants principaux (attestés), il y a des «bystanders ", c'est-à-dire des personnes qui assistent à la discussion sans y participer et même sans être perçues par les interactants (les cas extrême des eavesdroppers). Comment il en va des forums de discussion en ligne? En effet, si le statut de l'énonciateur du message est ici à peu près le même que dans la communica- 
tion directe (face-to-face), celui des instances de réception se modifient considérablement par rapport au modèle présenté ci-dessus. Vu que la discussion sur un forum exclut le contact visuel, qui est un paramètre crucial dans une interaction en direct, non seulement il est impossible de déterminer combien de personnes participe (ne serait-ce que passivement) à la discussion ${ }^{2}$, mais aussi qui est le destinataire explicite du post : sauf dans le cas où celuici contient le nick de l'usager précis ou la citation de ses paroles, les messages publiés dans un sujet s'adressent à l'ensemble des usagers du forum. De même, la distinction entre les overhearers et les eavesdroppers manque ici de pertinence, parce que les usagers passifs (bystanders) ne signalent aucunement leur présence aux interactants actifs; ils ne constituent qu'une " toile de fond " potentielle, d'ailleurs importante dans la mesure où tout usager actif est conscient du caractère public de ses posts.

Cette spécificité du modèle de la communication sur un forum de discussion en ligne n'est pas sans conséquence sur les perturbations que la structure de celle-là subit dans le cas de l'extension excessive du cadre temporel, décrite dans la section 2. Il est intéressant de noter que, dans la plupart des cas, le retour à un topic abandonné est initié par un usager qui jusqu'ici n'a pas participé à la discussion, alors que celle-ci l'a intéressé à tel point qu'il se décide à la relancer ( $c f$. l'exemple 1 qui confirme ce mécanisme). L'unicité du cadre participatif de l'interaction, indépendant de sa cohérence sémantique, se trouve par là rompue ou, du moins, considérablement modifiée. Comme nous l'avons signalé, l'usager qui reprend le sujet se réfère d'habitude au premier post, mais il arrive qu'il enchaîne sur une autre réplique de la discussion. En outre, même si des fois la conversation relancée s'étend sur plusieurs répliques consécutives, notre analyse d'une treintaine de topics de ce type démontre que, le plus souvent, la discussion renouvelée s'éteint vite (2-3 posts) ou même elle n'est pas reprise du tout, comme c'était justement le cas de l'exemple (1).

Quoi qu'il en soit, bien qu'une telle pratique soit marquée par rapport à la communication face-to-face, non seulement elle est bien fréquente sur les forums de discussion en ligne, mais elle peut être même considérée comme une démarche désirable ( $c f$. l'exemple 6 venant d'un forum sur les jeux de vidéo).

\footnotetext{
${ }^{2}$ Certains forums sont munis de compteurs de vues, cependant, ce paramètre n'est pas sûr (il rend compte plutôt de la popularité du sujet), étant donné que d'habitude, un usager (surtout actif) consulte la page plusieurs fois.
} 
(6) Total Warhammer 2 : Autant en emportent les skavens

Oor-tael: Tu sais que c'est pas comme ça que ça fonctionne un forum ? C'est assez bien foutu : si tu écris dans un vieux sujet, *POUF* il est dépoussiéré et remonte en tête de section!

Je t'assure que ça vaut le coup, c'est à essayer. Et ça évite de polluer les discussions.

(https://forum.canardpc.com/threads/95194-Total-Warhammer-2-Autant-enemportent-les-skavens/page193).

Il semble ainsi que pour les usagers eux-mêmes les paramètres formels (temps, structure, participants) de la discussion ne comptent pas beaucoup : ce qui est au centre, c'est l'unicité thématique du sujet traité. Pourtant, on ne saurait négliger que même celle-ci se trouve souvent perturbée, étant donné que le même sujet, repris deux ou trois ans plus tard, peut être vu sous un autre angle dans la dimension non seulement individuelle mais aussi sociale (changements au niveau de la loi, de l'usage, de la conscience sociale etc. selon le cas).

\section{CONCLUSION}

En général, le mode de développer une interaction en étendant à l'extrême le cadre temporel où elle s'inscrit conduit inévitablement à modifier ou même à perturber le modèle de l'interaction verbale entendue au sens traditionnel. Il est à observer que la perturbation d'un seul paramètre (cadre temporel) entraîne celle des autres (nombre des participants actifs, structure de l'interaction, notamment sa clôture). Il faut souligner néanmoins que ce phénomène n'a pas de caractère général, mais qu'il est strictement lié aux possibilités ouvertes par le logiciel du forum : la discussion continuée par de nouveaux participants qui interviennent activement après un certain temps dans un sujet abandonné n'est possible que dans ce type de communication virtuelle, où l'extension temporelle peut être pratiquement infinie (tant que le forum existe et le topic n'est pas fermé par les modérateurs).

\section{OUVRAGES CITÉES}

Cabasino, Francesca, editor. Du dialogue au polylogue, Approches linguistiques, socio-pragmatiques, littéraires. Actes du $3^{\mathrm{e}}$ Colloque international Do. Ri. F.-Università. CISU, 1998.

Calabrese, Laura, editor. L'internet, corpus sauvage. Nouvelles ressources, Nouveaux problèmes?, special issue of Le discours et la langue, vol. 21, 2010. 
Colin, Jean-Yves, and Florence Mourlhon-Dallies. "Du courrier des lecteurs aux forums de discussion sur l'internet : retour sur la notion de genre." Les Carnets du Cediscor, on line version: no. 8, 2004, pp. 113-140, http://journals.openedition.org/cediscor/707. 1 Nov. 2006. Accessed 20 Jan. 2020.

Francq, Pascal. "Le Web 2.0 et ses nouveaux corpus." Le discours et la langue, vol. 2.1., 2010/ 2011, pp. 105-109.

Marcoccia, Michel. "L'analyse conversationnelle des forums de discussion : questionnements méthodologiques." Les Carnets du Cediscor, on line version: no. 8, 2004, pp. 23-37, http://journals.openedition.org/cediscor/220. 1 Nov. 2006. Accessed 19 Jan. 2020.

Mourlhon-Dallies, Florence, Florimond Rakotonoelina, and Sandrine Reboul-Touré, editors. Les discours de l'internet: nouveaux corpus, nouveaux modèles?, special issue of Les Carnets du Cediscor, no. 8, 2004.

Münchow von, Patricia. "Le discours rapporté dans un forum de discussion sur l'internet." Les Carnets $d u$ Cediscor, on line version: no. 8, 2004, pp. 91-112, http://journals.openedition.org/ cediscor/702. 1 Nov. 2006. Accessed 29 Jan. 2020.

Pierozak, Isabelle. "Les corpus éléctroniques en sciences du langage : un eldorado ?" Le discours et la langue, vol. 2.1, 2010/2011, pp. 15-32.

Traverso, Véronique. “Aspects de négociation dans un polylogue.” Etudes romanes, no. 54, 2003, pp. 11-31.

Wołowska, Katarzyna. "Entre l'oral et l'écrit: la négociation d'identité et de position hiérarchique sur les forums d'Internet." Regards sur l'oral et l'écrit, edited by Krystyna Wróblewska-Pawlak, Jolanta Sujecka-Zając, and Elżbieta Pachocińska, Wydawnictwa UW, pp. 143-51.

\section{ZABURZENIE CZASOWYCH I AKTANCYJNYCH PARAMETRÓW INTERAKCJI SŁOWNEJ NA FORACH DYSKUSYJNYCH W INTERNECIE}

\section{Streszczenie}

Celem niniejszego artykułu jest opisanie kilku zaburzeń tradycyjnego modelu interakcji słownej w dyskusjach prowadzonych na forach internetowych. Analiza skupia się na opisie przypadków wątków dyskusyjnych prowadzonych na forach francuskojęzycznych, gdzie raz zakończona interakcja zostaje wznowiona i kontynuowana po dłuższym czasie (niekiedy po kilku miesiącach lub latach). Podjęcie dyskusji jest możliwe dzięki temu, że wątek jest bezterminowo otwarty, co pozwala na nawiązanie do dowolnej wypowiedzi w dowolnej chwili. Z punktu widzenia teoretycznego wpływa to na podstawowe parametry tradycyjnie rozumianej interakcji słownej, mianowicie na jej ramy czasowe (rozszerzenie czasu trwania dyskusji), strukturę (brak formuł zamykających dyskusję), a także na konfigurację aktancyjną uczestników dyskusji. Praktyka ta, choć niespotykana $\mathrm{w}$ interakcji bezpośredniej (face-to-face), jest całkowicie naturalna i często używana w dyskusjach na forach internetowych.

Słowa kluczowe: interakcja słowna; forum dyskusyjne; Internet; model komunikacji językowej; perturbacja. 


\section{PERTURBATION OF THE TIME AND PARTICIPATION FRAMEWORK OF THE INTERACTION ON ONLINE DISCUSSION FORUMS}

\section{S u m m a ry}

The aim of this paper is to describe some disturbances of the traditional model of verbal in interactions developed on online discussion forums. The analysis focuses on the description of topics raised on French forums, where the interaction, once completed, is continued after a long period. The discussion can be restarted since the topic is open indefinitely, which allows to refer to any post at any time. From a theoretical point of view, this affects the basic parameters of the traditionally understood verbal interaction, namely its time frame (extension of the duration of the discussion), its structure (lack of closing formulas), as well as its participation framework. This practice, although unusual in face-to-face interactions, is quite natural and frequent in discussions on online forums.

Key words: verbal interaction; discussion forum; Internet; model of verbal communication; disturbance. 